Pacific Journal of Mathematics

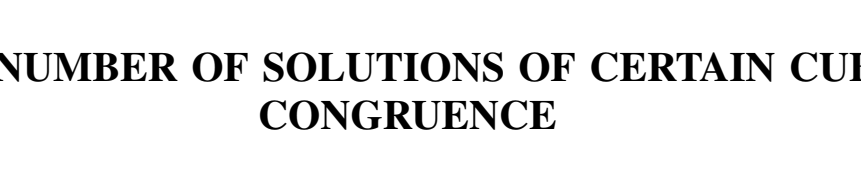




\title{
THE NUMBER OF SOLUTIONS OF CERTAIN CUBIC CONGRUENCES
}

\author{
ECKFORD COHEN
}

1. Introduction. In this paper we shall be concerned with cubic congruences of the form

$$
n \equiv a_{1} x_{1}^{3}+\cdots+a_{s} x_{s}^{3}
$$

$(\bmod m)$,

where $n$ is arbitrary, $m>1$, and the $a_{i}$ are integers prime to $m$. The number of sets of solutions $\left(x_{1}, \cdots, x_{s}\right)$ of (1.1), distinct modulo $m$, will be denoted by $N_{s}(n, m)$. Our discussion of $N_{s}(n, m)$ is limited to the cases $s=2$ and $s=3$; however, we emphasize that the method involved can be extended to arbitrary $s$.

Suppose that $m$ has the factorization $m=p_{1}^{\lambda_{1}}, \cdots, p_{l}^{\lambda_{l}}$ as a product of powers of distinct primes $p_{1}, \cdots, p_{l}$. Then it follows easily that

$$
N_{s}(n, m)=N_{s}\left(n, p_{1}^{\lambda}\right) \cdots N_{s}\left(n, p_{l}^{\lambda l}\right) .
$$

Thus the determination of $N_{s}(n, m)$ reduces to the problem of determining $N_{s}\left(n, p^{\lambda}\right)$ where $p$ is a prime. We accordingly limit ourselves to the case of a prime-power modulus $p^{\lambda}$.

If we denote by $t$ the largest integer $\leq \lambda$ such that $n \equiv 0\left(\bmod p^{t}\right)$, then one may write

$$
n=p^{t} \xi, \quad(\xi, p)=1, \quad 0 \leq t \leq \lambda .
$$

We observe, in case $\lambda>t$, that $\xi$ is uniquely determined $(\bmod p)$. Our main goal will be to obtain exact formulas for the number of solutions $N_{2}\left(n, p^{\lambda}, t\right)=N_{2}$ of

$$
n \equiv a x^{3}+b y^{3} \quad\left(\bmod p^{\lambda}\right),
$$

and the number of solutions $N_{3}\left(n, p^{\lambda}, t\right)=N_{3}$ of

$$
n \equiv a x^{3}+b y^{3}+c z^{3} \quad\left(\bmod p^{\lambda}\right),
$$

where $n$ is arbitrary of the form (1.3), and the following conditions are satisfied:

$$
p \equiv 1 \quad(\bmod 3), \quad a b c \neq \equiv 0 \quad(\bmod p) .
$$

The restriction $p \equiv 1(\bmod 3)$ is natural, since other primes are special in the case of cubic congruences.

The method of the paper is based on elementary properties of

Received March 5, 1954. This paper is based on research completed when the author was a member of the Institute for Advanced Study. 
finite exponential sums. These are listed for the cubic case as preliminary lemmas in $\S 2$. The principal formula for $N_{2}$ is contained in Theorem $1(\S 3)$ and the corresponding result for $N_{3}$ in Theorem $2(\S 4)$. Both results involve the pair of integers $(A, B)$, determined uniquely by the relations [7],

$$
4 p=A^{2}+27 B^{2}, \quad A \equiv 1(\bmod 3), \quad B>0 .
$$

However, in the special case $t \neq \equiv 0(\bmod 3)$, the value of $N_{2}$ is given explicity ( $\$ 3$, Corollary 2).

On the basis of these formulas, solvability criteria for (1.4) and (1.5) are developed in $\S 5$. In fact, it is shown in Theorem 5 that (1.5) is always solvable $\left(N_{3}>0\right)$. As for $N_{2}$, the following criterion is established: If $p \neq 7$, then (1.4) is insolvable if and only if $t \neq 0(\bmod$ $3), t<\lambda$, and $a$ and $b$ belong to different cubic character classes $(\bmod p)$. (For the exceptional case $p=7$, see the complete statement of the criterion in Theorem 6). Approximations to $N_{2}$ and $N_{3}$ are also given in $\S 5$ (Theorems 3 and 4 , respectively).

Regarding previous research on cubic congruences, we note the work of Gauss who evaluated $N_{2}$ in the case of a prime modulus $p$ [4]. More recently, Dickson determined $N_{3}$ for a prime modulus, with $a=b=c=1$ [3, p. 167]. In addition, Skolem [9] and Selmer [8] have considered such congruences in their treatment of cubic Diophantine equations. Some of these results were deduced by the author in an earlier note anticipating the present paper [2].

2. Notation and preliminary lemmas. The cubic Gauss sum $G(n, m)$ is defined by

$$
G(n, m)=\sum_{\mu(\bmod m)} \varepsilon\left(n^{3}, m\right),
$$

where the summation is over a complete residue system $(\bmod m)$, and $\varepsilon$ is defined for integral $\alpha$, by

$$
\varepsilon(\alpha, m)=e^{2 \pi i \alpha / m} .
$$

Expansion of $N_{s}(n, m)$ into a Fourier sum $[1, \S 5]$ reveals immediately the relation between $N_{s}(n, m)$ and the Gauss sum (2.1):

LEMma 1. The number of solutions of (1.1) is given by

$$
N_{s}(n, m)=\frac{1}{m} \sum_{\mu(\bmod m)} \varepsilon(n, m) \prod_{i=1}^{s} G\left(-a_{i} \mu, m\right) .
$$

We next note two reduction formulas for $G[6]$. 
Lemma 2.

$$
G\left(n m^{\prime}, m n^{\prime}\right)=m^{\prime} G(n, m)
$$

LeMMA 3. If $(\nu, p)=1$, then

$$
G\left(\nu, p^{k}\right)= \begin{cases}p^{2 j} & (k=3 j), \\ p^{2 j} G(\nu, p) & (k=3 j+1), \\ p^{2 j+1} & (k=3 j+2) .\end{cases}
$$

Closely related to $G\left(n, p^{k}\right)$ are the two Gauss-Kummer sums defined by

$$
\tau_{i}^{(k)}(n)=\sum_{\substack{\nu\left(\bmod p^{k}\right) \\(\nu, p)=1}} \chi^{i}(\nu) \varepsilon\left(n, p^{k}\right), \quad(i=1,2),
$$

where $\chi(\nu)$ and $\chi^{2}(\nu)$ denote the two non-principal cubic characters $(\bmod p)$, the summation being over a reduced residue system $\left(\bmod p^{k}\right)$. In order to differentiate between the two non-principal characters, we write

$$
\theta_{1}=\frac{1}{2}(A+3 B V-\overline{3}), \quad \theta_{2}=\overline{\theta_{1}}, \quad\left(\theta_{1} \theta_{2}=p\right),
$$

where $A$ and $B$ are defined by (1.7). Then one may define $\chi(\alpha)$, for integers $\alpha$ prime to $p$, to be that cube root of unity satisfying

$$
\chi(\alpha) \equiv \alpha^{(p-1) / 3} \quad\left(\bmod \theta_{1}\right) .
$$

The relation (2.8) is the cubic extension of the Euler criterion [5, p. 455]. In our discussion, the primitive cube roots of unity will be denoted by $\omega$ and $\omega^{2}$, with $\omega=\frac{1}{2}(-1+V-3)$.

We place further,

$$
\tau_{i}(n)=\tau_{i}^{(1)}(n), \quad \tau_{i}=\tau_{i}(1), \quad(i=1,2) .
$$

With this notation, we state the following reduction formula for $\tau_{i}^{(k)}(n)$.

LEMMA 4. If $k \geq 1$ and $i=1$ or 2 , then

$$
\tau_{i}^{(k)}(n)=\left\{\begin{array}{lc}
p^{k-1} \tau_{i}(\xi) & \left(n=p^{k-1} \xi, \quad(\xi, p)=1\right), \\
0 & \text { (otherwise) }
\end{array}\right.
$$

The important relation connecting $G(\nu, p), \tau_{1}(\nu)$, and $\tau_{2}(\nu)$ is contained in the following lemma.

Lemma 5. If $(\nu, p)=1$, then

$$
G(\nu, p)=\tau_{1}(\nu, p)+\tau_{2}(\nu, p) .
$$


The sums $\tau_{1}(\nu), \tau_{2}(\nu)$ have the following fundamental properties [5],

$$
\begin{gathered}
\tau_{1}(\nu)=\chi^{2}(\nu) \tau_{1}, \quad \tau_{2}(\nu)=\chi(\nu) \tau_{2}, \\
\tau_{1} \tau_{2}=p, \\
\tau_{1}^{3}=p \theta_{1}, \quad \tau_{2}^{3}=p \theta_{2},
\end{gathered}
$$$$
(\nu, p)=1 \text {, }
$$

$\theta_{1}$ and $\theta_{2}$ being defined by (2.7).

Corresponding to the principal character $(\bmod p)$, we have the familiar (Ramanujan) sum,

$$
C\left(n, p^{k}\right)=\sum_{\substack{\nu\left(\bmod p^{k}\right) \\(\nu, p)=1}} \varepsilon\left(n \nu, p^{k}\right),
$$

which has the evaluation $(k>0)$,

$$
C\left(n, p^{k}\right)=\left\{\begin{array}{cl}
p^{k-1}(p-1) & \left(p^{k} \mid n,\right. \\
-p^{k-1} & \left(p^{k-1} \mid n, p^{k} \nmid n\right), \\
0 & \left(p^{k-1} \nmid n\right) .
\end{array}\right.
$$

Also of importance in this paper are the functions,

$$
\begin{aligned}
& T(\alpha)=\frac{1}{p}\left(\chi^{2}(\alpha) \tau_{1}^{3}+\chi(\alpha) \tau_{2}^{3}\right), \\
& J(\alpha)= \begin{cases}A & (\chi(\alpha)=1), \\
\frac{1}{2}(9 h(\alpha) B-A) & (\chi(\alpha) \neq 1),\end{cases}
\end{aligned}
$$

where $h(\alpha)$ is defined for cubic non-residues $\alpha(\bmod p)$ by

$$
h(\alpha)=1 \quad \text { or }-1
$$

according as $\chi(\alpha)=\omega$ or $\omega^{2}$.

Application of (2.13) gives

LEMMA 6.

$$
T(\alpha)=J(\alpha)
$$

The following notation will be needed.

$$
\begin{aligned}
& q=\left[\frac{t-1}{3}\right], \quad r=\left[\begin{array}{c}
t \\
3
\end{array}\right], \quad s=\left[\begin{array}{c}
t-2 \\
3
\end{array}\right], \\
& Q=\left[\frac{\lambda-1}{3}\right], \quad R=\left[\frac{\lambda}{3}\right], \quad S=\left[\frac{\lambda-2}{3}\right] \text {, }
\end{aligned}
$$

where $[\beta]$ indicates the largest integer $\leq \beta$; and for $i=0,1,2$,

$$
L_{i}(t)= \begin{cases}1 & (t \equiv i(\bmod 3), \quad t<\lambda), \\ 0 & (\text { otherwise }) .\end{cases}
$$


3. The number of solutions of (1.4). In this section we use the notation,

$$
\zeta=a b \xi,
$$

where $\xi$ is defined by (1.3), and

$$
\eta=\chi(a) \chi^{2}(b)+\chi(b) \chi^{2}(a)=2 \text { or }-1,
$$

according as $\chi(a)=\chi(b)$ or $\chi(a) \neq \chi(b)$.

The main result on (1.4) is contained in

THEOREM 1. The number of solutions of (1.4) is given by

$$
\begin{aligned}
N_{2}\left(n, p^{\lambda}, t\right)=p^{\lambda-1}\{ & p^{r} J(\zeta) L_{0}(t)+p^{q+1} r\left(1-L_{0}(t)\right) \\
& \left.+p^{r+1}\left(1-L_{2}(t)\right)+p^{s+1}\left(1-L_{1}(t)\right)-(\eta+1)\right\},
\end{aligned}
$$

where $t$ is defined by (1.3), $J$ by (2.17), $q, r, s$ by (2.20), the $L_{i}(t)$ by (2.22), and $\zeta, \eta$ by (3.1) and (3.2) respectively.

Proof. By Lemma 1 it follows immediately that

$$
N_{2}=\frac{1}{p^{\lambda}} \sum_{\left.\bmod p^{\lambda}\right)} \varepsilon\left(n \mu, p^{\lambda}\right) G\left(-a \mu, p^{\lambda}\right) G\left(-b \mu, p^{\lambda}\right) .
$$

The residue system $\mu\left(\bmod p^{\lambda}\right)$ may be assumed to be the set $\mu=\nu p^{\lambda-k}$ where $k$ ranges over the values $0 \leq k \leq \lambda$, and for each $k$, ranges over a reduced residue system $\left(\bmod p^{k}\right)$. Thus $(3.4)$ becomes, using $(2.4)$,

$$
N_{2}=p^{\lambda} \sum_{k=0}^{\lambda} \frac{1}{p^{2 k}} \sum_{\substack{\nu(\bmod \\(\nu, p)=1}} \varepsilon\left(\nu n, p^{k}\right) G\left(-a_{\nu}, p^{h}\right) G\left(-b \nu, p^{k}\right) .
$$

We now break up the $k$ summation according as $k \equiv 1,0$, or $2(\bmod 3)$, and apply Lemma 3 to obtain

$$
N_{2}=U_{1}+U_{2}+U_{3},
$$

where

$$
\begin{aligned}
& U_{1}=p^{\lambda-2} \sum_{j=0}^{Q} \frac{1}{p^{2 J}} \sum_{\substack{\left(\bmod p^{3 j+1}\right) \\
(\nu, p)=1}} \varepsilon\left(\left\llcorner n, p^{3 j+1}\right) G\left(-a_{\nu}, p\right) G(-b \nu, p)\right. \text {, } \\
& U_{2}=p^{\lambda} \sum_{j=0}^{R} \frac{1}{p^{2 j}} C\left(n, p^{3 j}\right), \quad U_{3}=p^{\lambda-2} \sum_{j=0}^{S} \frac{1}{p^{2 j}} C\left(n, p^{3 j+2}\right) .
\end{aligned}
$$

Applying Lemma 5 and (2.11) to (3.7), and expanding, $U_{1}$ may be written 


$$
U_{1}=U_{11}+U_{12}+U_{13}
$$

where

$$
\begin{aligned}
& U_{11}=p^{\lambda-2} \chi^{2}(a b) \tau_{1}^{2} \sum_{j=0}^{Q} \frac{1}{p^{2 j}} \tau_{1}^{\left({ }^{3 j+1}\right)}(n), \\
& U_{12}=p^{\lambda-2} \chi(a b) \tau_{2}^{2} \sum_{j=0}^{Q} \frac{1}{p^{2 j}} \tau_{2}^{(3 j+1)}(n), \\
& U_{13}=p^{\lambda-2} \tau_{1} \tau_{2} \eta \sum_{j=0}^{Q} \frac{1}{p^{2 j}} C\left(n, p^{3 j+1}\right) .
\end{aligned}
$$

Application of (2.11) and Lemmas 4 and 6 to $U_{11}$ and $U_{12}$ gives

$$
U_{11}+U_{12}=p^{\lambda-1+r} J(\zeta) L_{0}(t),
$$

while $U_{13}$ becomes, on the basis of (2.12) and (2.15),

$$
U_{13}=p^{\lambda-1} \eta\left\{p^{q+1}\left(1-L_{0}(t)\right)-1\right\} \text {. }
$$

Also, using (2.15) and summing, we get

$$
U_{2}=p^{\lambda+r}\left(1-L_{2}(t)\right), \quad U_{3}=p^{\lambda-1}\left\{p^{s+1}\left(1-L_{1}(t)\right)-1\right\} .
$$

The theorem follows on combining (3.6), (3.9), (3.10), (3.11), and (3.12).

Three main cases of Theorem 1 are distinguished according as, (i) $\lambda>t, t \equiv 0(\bmod 3)$, (ii) $\lambda>t, t \neq \equiv(\bmod 3)$, or (iii) $\lambda=t \quad(n=0)$. Corresponding to these cases, one may deduce the following corollaries from (3.3).

CoRollary 1. If $\lambda>t=3 e$, then

$$
N_{2}\left(n, p^{\lambda}, 3 e\right)=p^{\lambda-1}\left\{p^{e}(J(\zeta)+p+1)-\eta-1\right\} \text {. }
$$

COROLlaRY 2. If $\lambda>t \neq 0(\bmod 3)$, then

$$
N_{2}\left(n, p^{\lambda}, t\right)=p^{\lambda-1}\left(p^{e+1}-1\right)(\eta+1),
$$

where $t=3 e+1$ or $3 e+2$, according as $t \equiv 1$ or $2(\bmod 3)$.

Corollary 3. $(n=0)$. If $\lambda=t=3 e+j,(j=0,1,2)$, then

$$
N_{2}\left(n, p^{\lambda}, \lambda\right)=p^{\lambda-1}\left\{(\eta+1)\left(p^{e+\gamma}-1\right)+p^{e+j+1-2 \gamma}\right\},
$$

where $r=0$ or 1 according as $t \equiv 0$ or $t \neq \equiv 0(\bmod 3)$.

4. The number of solutions of (1.5). The elements of the set $(a, b, c, \xi)=H$ may be distributed among the three cubic character classes $(\bmod p)$ in essentially four different ways. These four distributions, denoted by $H_{1}, H_{2}, H_{3}$, and $H_{4}$, are defined as follows: $\left(H_{1}\right)$ 
Every class contains at least one element of $H ;\left(H_{2}\right)$ One class contains two elements of $H$ and a second class contains the other two; $\left(H_{3}\right)$ One class contains three elements of $H$ but not all four; $\left(H_{1}\right)$ All four elements lie in the same class.

Using this notation we define the function,

$$
\delta(H)=0,3,-3 \text {, or } 6,
$$

according as the elements of $H$ have a distribution of type $H_{1}, H_{2}, H_{3}$, or $H_{4}$.

We will also make use of the following notation:

$$
\theta=a b c ; \quad \eta_{1}=\eta_{1}(a, b, c)=\chi(a) \chi^{2}(b c)+\chi(b) \chi^{2}(a c)+\chi(c) \chi^{2}(a b), \quad \eta_{2}=\bar{\eta}_{1},
$$

$\bar{\eta}_{1}$ denoting the complex conjugate of $\eta_{1}$ :

$$
\Delta(H)=\eta_{1} \chi(\xi)+\eta_{2} \chi^{2}(\xi)
$$

On the basis of the above notation, one may deduce

LEMMA 7.

$$
\Delta(H)=\delta(H) .
$$

We now state the main theorem for $N_{3}\left(n, p^{\lambda}, t\right)$.

THEOREM 2. The number solutions $r f(1.5)$ is given by

$$
\begin{aligned}
N_{3}\left(n, p^{\lambda}, t\right)=p^{2 \lambda-2}\{ & {\left[(p-1)(q+1)-L_{0}(t)\right] J(\theta)+p \delta(H) L_{0}(t) } \\
& \left.-L_{1}(t)-p L_{2}(t)+(p-1)(p r+s+1)+p^{2}\right\},
\end{aligned}
$$

where $\delta(H)$ is defined by (4.1), $\theta$ by (4.2), and the rest of the notation has the same meaning as in Theorem 1.

Proof. As in the proof of Theorem 1, we may express $N_{3}$ as a Fourier sum and apply Lemmas 2 and 3 to obtain

$$
N_{3}=V_{1}+V_{2}+V_{3},
$$

where

$$
\begin{gathered}
V_{1}=p^{2 \lambda-3} \sum_{j=0}^{Q} \sum_{p^{3 j}}^{1} \sum_{\substack{\left(\bmod p^{3 j+1}\right) \\
(\nu, p)=1}} \varepsilon\left(\nu n, p^{3 j+1}\right) G\left(-a_{\nu}, p\right) G(-b \nu, p) G(-c \nu, p), \\
V_{2}=p^{2 \lambda} \sum_{j=0}^{R} \frac{1}{p^{3 j}} C\left(n, p^{3 j}\right), \quad V_{3}=p^{2 \lambda-3} \sum_{j=0}^{S} \frac{1}{p^{3 j}} C\left(n, p^{3 j+2}\right) .
\end{gathered}
$$

Application of Lemma 5 and (2.11) to (4.7) yields

$$
V_{1}=V_{11}+V_{12}+V_{13}
$$


where

$$
\begin{aligned}
& V_{11}=p^{2 \lambda-2} T(\theta) \sum_{j=0}^{Q} \frac{1}{p^{3 j}} C\left(n, p^{3 j+1}\right), \\
& V_{12}=p^{2 \lambda-3} \tau_{1}^{2} \tau_{2} \eta_{1} \sum_{j=0}^{Q} \frac{1}{p^{3 j}} \tau_{2}^{(3 j+1)}(n), \\
& V_{13}=p^{2 \lambda-3} \tau_{1} \tau_{2}^{2} \eta_{2} \sum_{j=0}^{Q} \frac{1}{p^{3 j}} \tau_{1}^{(3 j+1)}(n) .
\end{aligned}
$$

Using (2.15) and Lemma 6 in case of $V_{11}$, one obtains

$$
V_{11}=p^{2 \lambda-2} J(\theta)\left\{(p-1)(q+1)-L_{0}(t)\right\} .
$$

$V_{12}$ and $V_{13}$ may be transformed by (2.11), (2.12), and Lemmas 4 and 7 , to give

$$
V_{12}+V_{13}=p^{2 \lambda-1} \delta(H) L_{0}(t) .
$$

As for $V_{2}$ and $V_{3}$, application of (2.15) gives

$$
\begin{aligned}
& V_{2}=p^{2 \lambda-2}\left\{p^{2}+p r(p-1)-p L_{2}(t)\right\}, \\
& V_{3}=p^{2 \lambda-2}\left\{(p-1)(s+1)-L_{1}(t)\right\} .
\end{aligned}
$$

Combination of the results in (4.6) and formulas (4.10) through (4.13) leads to the theorem.

Corresponding to the corollaries of Theorem 1, we may deduce the following results as special cases of Theorem 2 .

CoROLlary 1. If $\lambda>t=3 e$, then

$$
N_{3}\left(n, p^{\lambda}, 3 e\right)=p^{2 \lambda-2}\left\{(p e-e-1) J(\theta)+e\left(p^{2}-1\right)+p^{2}+p \delta(H)\right\} \text {. }
$$

CoROLlaRY 2. If $\lambda>t \neq 0(\bmod 3)$, then

$$
N_{3}\left(n, p^{\lambda}, t\right)=p^{2 \lambda-2}(p-1)(e+1)(J(\theta)+p+1),
$$

where $t=3 e+1$ or $3 e+2$.

Corollary $3(n=0)$. If $\lambda=t$, then

$$
N_{3}\left(n, p^{\lambda}, \lambda\right)=p^{2 \lambda-2}\left\{(p-1)\left[J(\theta)\left(e+\mu_{1}\right)+e(p+1)+\mu_{2}\right]+p^{2}\right\},
$$

where $\mu_{1}=\mu_{2}=0$ if $t=3 e>0 ; \mu_{1}=1, \mu_{2}=0$ if $t=3 e+1$, and $\mu_{1}=\mu_{2}=1$ if $t=3 e+2$.

5. Solvability criteria. First we establish some bounds for $N_{\Sigma}$ and $N_{3}$. To do this, note by Definition (1.7) that $|A|<2 \sqrt{p}$, and by a simple process of maximalization, that $|9 h(\alpha) B-A|<4 \sqrt{p},(h(\alpha)= \pm 1)$. Thus we have 
LEMMA 8.

$$
|J(\alpha)|<2 \vee p
$$

By means of this Lemma and Corollary 1 of $\S 3$, we get the following estimate for $N_{2}\left(n, p^{\lambda}, 3 e\right)$.

Theorem 3. If $\lambda>t=3 e$, then

$$
p^{e}(p+1-2 \sqrt{p})-\eta-1<\frac{N_{2}}{p^{\lambda-1}}<p^{e}(p+1+2 \sqrt{ } p)-\eta-1 .
$$

Similarly, we may deduce bounds for $N_{3}$ on the basis of Corollaries 1 and 2 of $\S 4$.

Theorem 4. If $\lambda>t$, then in case $t=3 e$,

$$
\begin{gathered}
p^{2}+e\left(p^{2}-1\right)-2(p e-e-1) \sqrt{p}+p \delta(H)<p^{2(1-\lambda)} N_{3} \\
<p^{2}+e\left(p^{2}-1\right)+2(p e-e-1) \sqrt{p}+p \delta(H),
\end{gathered}
$$

and in case $t=3 e+1$ or $3 e+2$,

$$
p+1-2 \vee p<\frac{p^{2(1-\lambda)} N_{3}}{(p-1)(e+1)}<p+1+2 \vee p .
$$

We are now in a position to establish precise criteria for the solvability of (1.4) and (1.5).

THEOREM 5. The congruence (1.5) has a solution for every integer $n$.

Proof. To prove this theorem it suffices to show that the lower bounds in (5.3) and (5.4) are positive. This follows immediately in the case of (5.4). Rewriting the lower bound in (5.3) in the form,

$$
e p^{3 / 2}(\sqrt{p}-2)+e(2 \sqrt{p}-1)+p\left(p+2 p^{-1 / 2}+\delta\right),
$$

and remembering that the minimal values of $p, \delta(H)$, and $e$ are $p=7$, $\delta=-3$, and $e=0$, we see that $N_{3}>0$ also in the case $\lambda>t \equiv 0(\bmod 3)$.

THEOREM 6. The congruence (1.4) has no solution if and only if either $t \neq \equiv 0(\bmod 3), t<\lambda$, and $\chi(a) \neq \chi(b)$, or if $p=7, t=0, \chi(a)=\chi(b)$ and $\zeta=a b \xi \equiv \pm 3(\bmod 7)$.

Proof. If $\lambda>t \neq 0(\bmod 3)$, it follows directly from Corollary 2 of $\S 3$, that $N_{2}=0$ if and only if $\eta=-1(\chi(a) \neq \chi(b))$. In the remainder of the proof we suppose, therefore, that $\lambda>t \equiv 0(\bmod 3)$. Now the 
lower bound in (5.2) is positive in case $\eta=-1$ and also in case $\eta=2$, $e>0$. In the remaining case $(\eta=2, e=0)$, the lower bound is $p-2-$ $2 \checkmark p$, which is positive if $p>7$. But if $p=7, e=0, \eta=2$, then substitution in (3.13) shows that $N_{2}=0$ if and only if $\chi(\zeta)=\omega^{2}$, which implies that $\zeta \equiv \pm 3(\bmod 7)$.

As a corollary of Theorem 6, we have the following result [8], [9]:

CoROLlaRY (Skolem-Selmer). If $p \nmid a b c$, then the congruence

$$
a x^{3}+b y^{3}+c z^{3} \equiv 0 \quad\left(\bmod p^{\lambda}\right)
$$

always has a non-trivial solution $(x, y, z$ not all $\equiv 0(\bmod p))$.

Proof. With $z=1, c=-n$, Theorem 6 shows that (5.5) has a nontrivial solution $(X, Y, 1)$ unless $p=7, \chi(a)=\chi(b)$. In the latter case, however, there exists a solution $(X, 1,0)$, because an integer $\alpha$ is a cubic residue $\left(\bmod p^{\lambda}\right)$ if and only if it is a residue $(\bmod p)$.

\section{REFERENCES}

1. Eckford Cohen, Rings of arithmetic functions, Duke Math. J., 19 (1952), 115-129.

2. ___ Representations by cubic congruences, Proc. Nat. Acad. Sci., 39 (1953), 119121.

3. L.E. Dickson, Congruences involving only e-th powers, Acta Arithmetica, 1 (1935), 162167.

4. C.F. Gauss, Werke, I, 445-449.

5. Helmut Hasse, Vorlesungen über Zahlentheorie, Berlin, 1950, 453-455.

6. Edmund Landau, Vorlesungen über Zahlentheorie, I, Leipzig, 1927, 280-302.

7. G.B. Mathews, Theory of Numbers, Part I, reprinted New York, 1927, p. 222.

8. Ernst Selmer, The Diophantine equation $a x^{3}+b y^{3}+c z^{3}=0$, Acta Math., 85 (1951), 215223.

9. Th. Skolem, Unlösbarkeit von Gleichungen, deren entsprechende Kongruenz für jeden Modul lösbar ist, Avh. Norske Vid. Akad. Oslo, I. 4 (1942), 6-14.

The University of South Carolina 
.././. ./FrontMatter/paper .pdf 


\section{Pacific Journal of Mathematics}

Nesmith Cornett Ankeny and Theodore Joseph Rivlin, On a theorem of S.

Bernstei........................................ 849

Louis Auslander, The use of forms in variational calculation .......... 853

Paul Civin, Abstract Riemann sum . .......................... 861

Paul Civin, Some ergodic theorems involving two operator ............ 869

Eckford Cohen, The number of solutions of certain cubic congruence .... . 877

Richard M. Cohn, Specializations over difference field .............. 887

Jean Dieudonné, Pseudo-discriminant and Dickson invarian . . ......... 907

Ky Fan, A comparison theorem for eigenvalues of normal matrice ........ 911

Richard P. Gosselin, On the convergence behaviour of trigonometric interpolating polynomial ........................... 915

Peter K. Henrici, On generating functions of the Jacobi polynomial . . . . . . . 923

Meyer Jerison, An algebra associated with a compact grou ............ 933

Wilhelm Magnus, Infinite determinants associated with Hill's equatio . . . . . 941

G. Power and D. L. Scott-Hutton, The slow steady motion of liquid past a semi-elliptical bos................................. 953

Lyle E. Pursell, An algebraic characterization of fixed ideals in certain function ring .................................... 963

C. T. Rajagopal, Additional note on some Tauberian theorems of O. Szás . . 971 Louis Baker Rall, Error bounds for iterative solutions of Fredholm integral

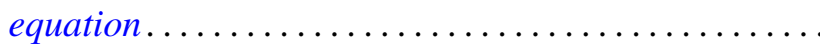

Shigeo Sasaki and Kentaro Yano, Pseudo-analytic vectors on

pseudo-Kählerian manifold ......................

Eugene Schenkman, On the tower theorem for finite group

P. Stein and John E. L. Peck, On the numerical solution of Poisson's equation over a rectangl ........................

Morgan Ward, The mappings of the positive integers into themselves which preserve divisio .

Seth Warner, Weak locally multiplicatively-convex algebra 1025

Louis Weisner, Group-theoretic origin of certain generating function .... 